\title{
Improved Sampling and Free Energy Estimates for Antibiotics Permeation through Bacterial Porins
}

\author{
Abhishek Acharya, Jigneshkumar Dahyabhai Prajapati, and Ulrich \\ Kleinekathöfer* \\ Department of Physics and Earth Sciences, Jacobs University Bremen, 28759 Bremen, \\ Germany
}

E-mail: u.kleinekathoefer@jacobs-university.de 


\section{S1 FES estimation procedures}

\section{S1.1 Unified Free Energy Dynamics with WTmetaD bias along the principal $\mathrm{CV}$}

For FES estimation along the CV vector space $s$ we use the procedure described previously in connection with the TASS method. ${ }^{1}$ Briefly, the Tiwary-Parrinello scheme ${ }^{2}$ is used to first obtain the probability distribution reweighted for the metadynamics bias applied along the auxillary $\mathrm{CV} s_{1}$

$$
\widetilde{P}(\boldsymbol{s})=\frac{\sum_{t} \delta\left(s_{\alpha}(t)-s_{\alpha}\right) \exp \left(\frac{V\left(s_{1}, t\right)-c(t)}{k_{B} \widetilde{T}}\right)}{\sum_{t} \exp \left(\frac{V\left(s_{1}, t\right)-c(t)}{k_{B} \widetilde{T}}\right)}
$$

where $c(t)$ is given by

$$
c(t)=\frac{1}{\widetilde{\beta}} \log \frac{\int d s_{1} \exp \left(\widetilde{\beta} \gamma V\left(s_{1}, t\right)\right)}{\left.\int d s_{1} \exp \left(\widetilde{\beta}(\gamma-1) V\left(s_{1}, t\right)\right)\right)}
$$

Here, $V\left(s_{1}, t\right)$ is a time-dependent Gaussian potential bias deposited along the auxillary $\mathrm{CV}$ $s_{1}$ and the bias factor $\gamma$ is related to the metadynamic bias tuning factor $\Delta T$ by the relation

$\gamma=\frac{(T+\Delta T)}{\Delta T}$. Note that the temperature in Eqns. 1 and 2 is $\widetilde{T}$ - the temperature of the extended system. In the next step, we appropriately scale $\widetilde{P}(s)$ using the following relation

$$
P_{u}(s) \propto \widetilde{P}(s)^{(\beta / \widetilde{\beta})}
$$

where $P_{u}(\boldsymbol{s})$ is the unbiased probability.

Finally, the free energy can be determined using the relation

$$
F(s)=-\frac{1}{\beta} \ln P_{u}(s)+f
$$

where $f$ is some constant. 


\section{S1.2 Temperature accelerated sliced sampling}

In the TASS scheme, ${ }^{1}$ a harmonic bias potential and a time-dependent metadynamics bias is applied along the auxiliary $\mathrm{CVs} s_{1}$ and $s_{2}$, respectively, while motions along other orthogonal CVs are enhanced by uniform thermal fluctuations using a high temperature bath. In the original weighted histogram analysis method (WHAM) based approach, for $h$ umbrella windows we first obtain the probabilities reweighted for the metadynamics bias applied along $\mathrm{CV} s_{2}$ using the Tiwary-Parrinello scheme. ${ }^{2}$

$$
\widetilde{P_{h}}\left(\boldsymbol{s}^{\prime}\right)=\frac{\sum_{t} \delta\left(s_{\alpha}(t)-s_{\alpha}^{\prime}\right) \exp \left(\frac{V\left(s_{2}, t\right)-c(t)}{k_{B} \widetilde{T}}\right)}{\sum_{t} \exp \left(\frac{V\left(s_{2}, t\right)-c(t)}{k_{B} \widetilde{T}}\right)}
$$

where $c(t)$ is given by

$$
c(t)=\frac{1}{\widetilde{\beta}} \log \frac{\int d s_{2} \exp \left(\widetilde{\beta} \gamma V\left(s_{2}, t\right)\right)}{\left.\int d s_{2} \exp \left(\widetilde{\beta}(\gamma-1) V\left(s_{2}, t\right)\right)\right)} .
$$

Here, $V\left(s_{2}, t\right)$ denotes the metadynamics bias potential deposited along the CV $s_{2}$. For the case of TASS without metadynamics bias, the normalized probability distributions $\widetilde{P_{h}}\left(\boldsymbol{s}^{\prime}\right)$ can be directly calculated by histogramming.

Subsequently, the individual probabilities need to be reweighted for the harmonic potential bias. The probabilities $\widetilde{P_{h}}\left(s^{\prime}\right)$ are reweighted and patched together using WHAM to obtain the combined probability distribution bereft of the umbrella biases. The following WHAM equations are solved in a self-consistent manner to obtain $\widetilde{P}(\boldsymbol{s})$.

$$
\begin{aligned}
\widetilde{P}(s) & =\frac{\sum_{h=1}^{m} n_{h} \widetilde{P}_{h}(s)}{\sum_{h=1}^{m} n_{h} \exp \left(-\widetilde{\beta}\left[W_{h}\left(s_{1}\right)-f_{h}\right]\right)} \\
\exp \left(-\widetilde{\beta} f_{h}\right) & =\int d s P(s) \exp \left[-\widetilde{\beta} W_{h}\left(s_{1}\right)\right]
\end{aligned}
$$

Here, $n_{h}$ denotes the number of samples and $W_{h}\left(s_{1}\right)$ the harmonic bias potential correspond- 
ing to umbrella window $h$. The final unbiased distribution $P_{u}(s)$ at system temperature $T$ can then be calculated using the following relation.

$$
P_{u}(s) \propto \widetilde{P}(s)^{(\beta / \widetilde{\beta})} .
$$

Finally, the free-energy can be determined from the unbiased probability using Eq. (4). However, this WHAM-based procedure may perform poorly in certain cases. It requires numerous umbrellas simulations for a good overlap which is necessary to ensure proper convergence. Thus, it is often slow to converge and may provide poor estimates for the PMF.

In this work, we have employed a alternate mean force based procedure developed by Nair and coworkers that circumvents the use of WHAM for the reconstruction of high- dimensional FES. ${ }^{3}$ Briefly, the method calculates the high-dimensional FES using the relation

$$
F(\boldsymbol{s})=F_{1}\left(s_{1}\right)+\Delta F_{s_{1}}\left(s_{2}, \ldots, s_{n}\right) .
$$

The first term $F_{1}$ on the right is the free-energy along the auxiliary $\mathrm{CV} s_{1}$ that was sampled using the harmonic umbrella bias. For the TASS Hamiltonian without a metadynamics bias, $F_{1}$ can be calculated in a straightforward manner using

$$
F_{1}\left(s_{1}\right)=\int^{s_{1}}\left\langle k_{h}\left(s_{1}-s_{1}^{h}\right)\right\rangle_{s_{1}} d s_{1},
$$

where $\left\{k_{h}\right\}$ denotes the harmonic spring constants used for the umbrella biases. This term estimates the 1D free energy along the principal umbrella CV by calculating the average force at each restraint position along $s_{1}$ to obtain the corresponding gradients. The force along $s_{1}$ is then integrated to obtain the free energy $F_{1}$ at all $s_{1}$ positions. Free-energy values at intermediate points are generated using interpolation. The second term on the right of Eq. 10 denotes the $n-1$ dimensional free energy at $s_{1}$. This can be calculated using 
the relation

$$
\Delta F\left(s_{2}, \ldots, s_{n}\right)=-\frac{1}{\widetilde{\beta}} \ln P_{s_{1}}\left(s_{2}, \ldots, s_{n}\right)
$$

where $P_{s_{1}}$ denotes the probability distribution corresponding to each umbrella window obtained by histogramming. Finally, the two terms in Eq. 10 can be combined to obtain the high-dimensional FES. Thus, the method essentially uses the 1D FES along $s_{1}$ to appropriately reweight the high-dimensional distributions obtained from individual umbrella simulations.

\section{S2 Preliminaries and convergence for independent WT-}

\section{metaD calculations}
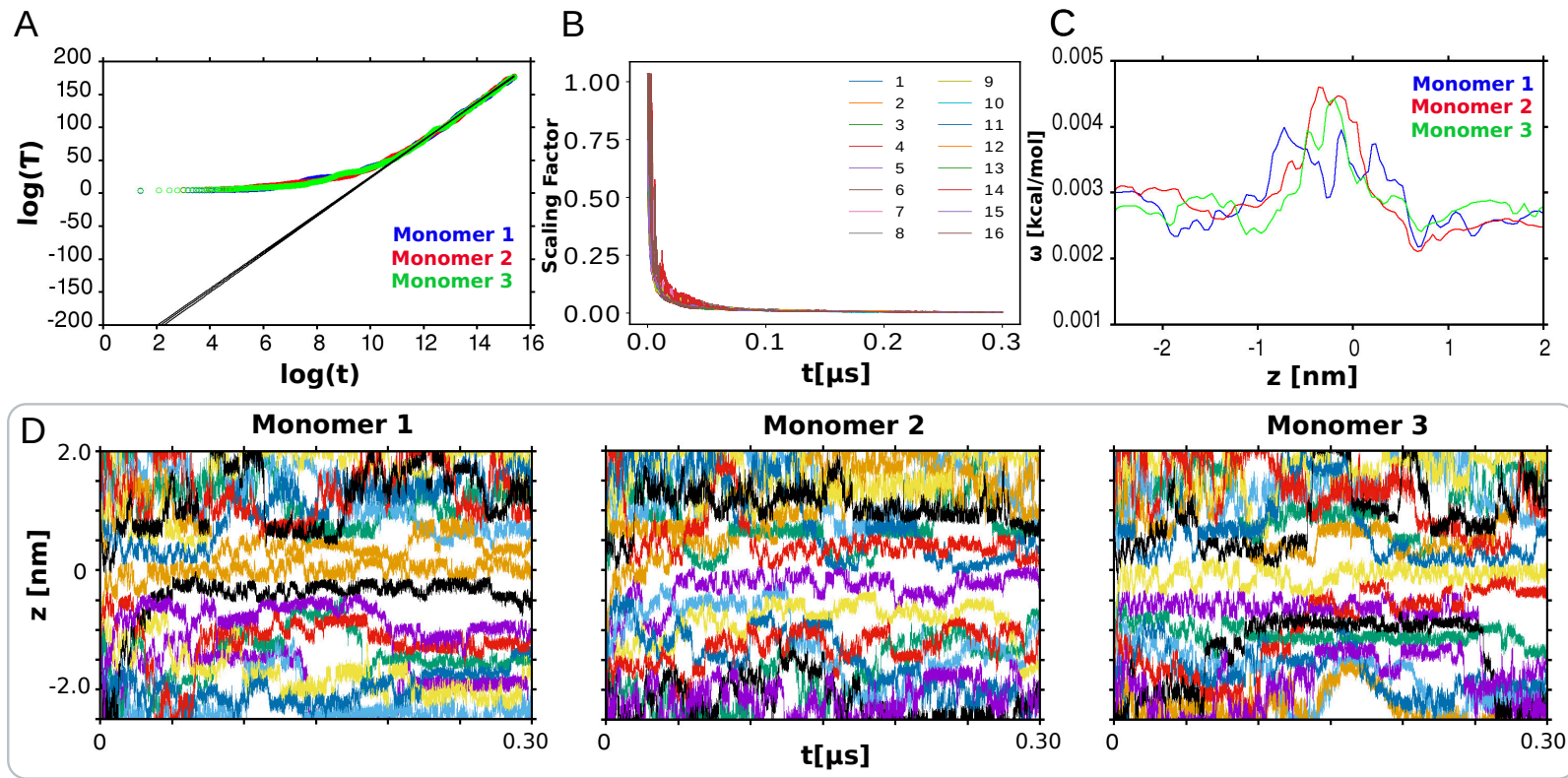

Figure S1: (A) Plot for the scaled time $\tau$ vs. simulation time $t$ on a log-log scale for independent WTmetaD simulations of the three OmpF monomers. (B) The decay of the height scaling factor. A representative plot obtained for calculations of monomer 1 is provided. (C) Plot depicting the scaled heights of the last deposited bias along the $z \mathrm{CV}$ space for the three independent simulations. (D) Time evolution of the $\mathrm{CV} z$ position for the 16 walkers as observed in independent runs. Each walker was propagated for $300 \mathrm{~ns}$ giving a total simulation time of $4.8 \mu$ s for each run. 
As shown in Figure S1, panel A, the scaled time $\tau$ versus the simulation time $t$ on a $\log$-log scale shows that for WTmetaD simulations, $\log (\tau)$ achieves linearity with respect to $\log (t)$ satisfying the asymptotic relation $\log (\tau) \propto \gamma \log (t)$, in the limit $t \rightarrow \infty$. The slope of the linear fit to this plot gives the bias factor, $\gamma \approx 30$, so the above relations hold true. In addition, panel B in Figure S1 depicts the evolution of the Gaussian bias scaling factor for each of the walkers. The scaling factor for the hills decays to zero with time which indicates that after an initial period, the bias deposition becomes sufficiently slow such that the system achieves equilibrium. Together these plots show that the simulations attained the quasistationary limit. As mentioned in the main text, in these simulations we used 9 and 7 walkers starting on the EC and PP side, respectively, to ensure even sampling of the CV space. A plot of the last deposited Gaussian heights along CV $z$ shows even sampling in both EC and PP region, as evident from similar hill heights, thereby validating our choice of an unsymmetrical walker distribution as can be seen in Figure S1, panel C. Slightly higher values (difference of $<0.002 \mathrm{kcal} / \mathrm{mol}$ ) in the CR indicate that this region has less sampling compared to the vestibule regions. The difference is, however, marginal and as such these values indicate even sampling in all regions of the CV space. Figure S1, bottom row, depicts the time-evolution of the $\mathrm{CV} z$ for individual walkers from the three MWWTmetaD simulations. Although, the entire CV space is explored in all the simulations, we find that none of the walkers was able to cross the CR to the other side. Moreover, there is a lack of diffusive sampling as many walkers appear to be stuck in various regions of the potential energy landscape. These problems have been consistently observed in simulations with large solutes, presumably occurring due to insufficient sampling of the rotational degrees of freedom for the solute. Even though the above observations are indicative of problems in sampling, from the time-evolution of the individual 1D FES, it appears that the individual free energy estimates have achieved convergence within $4 \mu$ s of simulation time (see Figure S2, top row). 

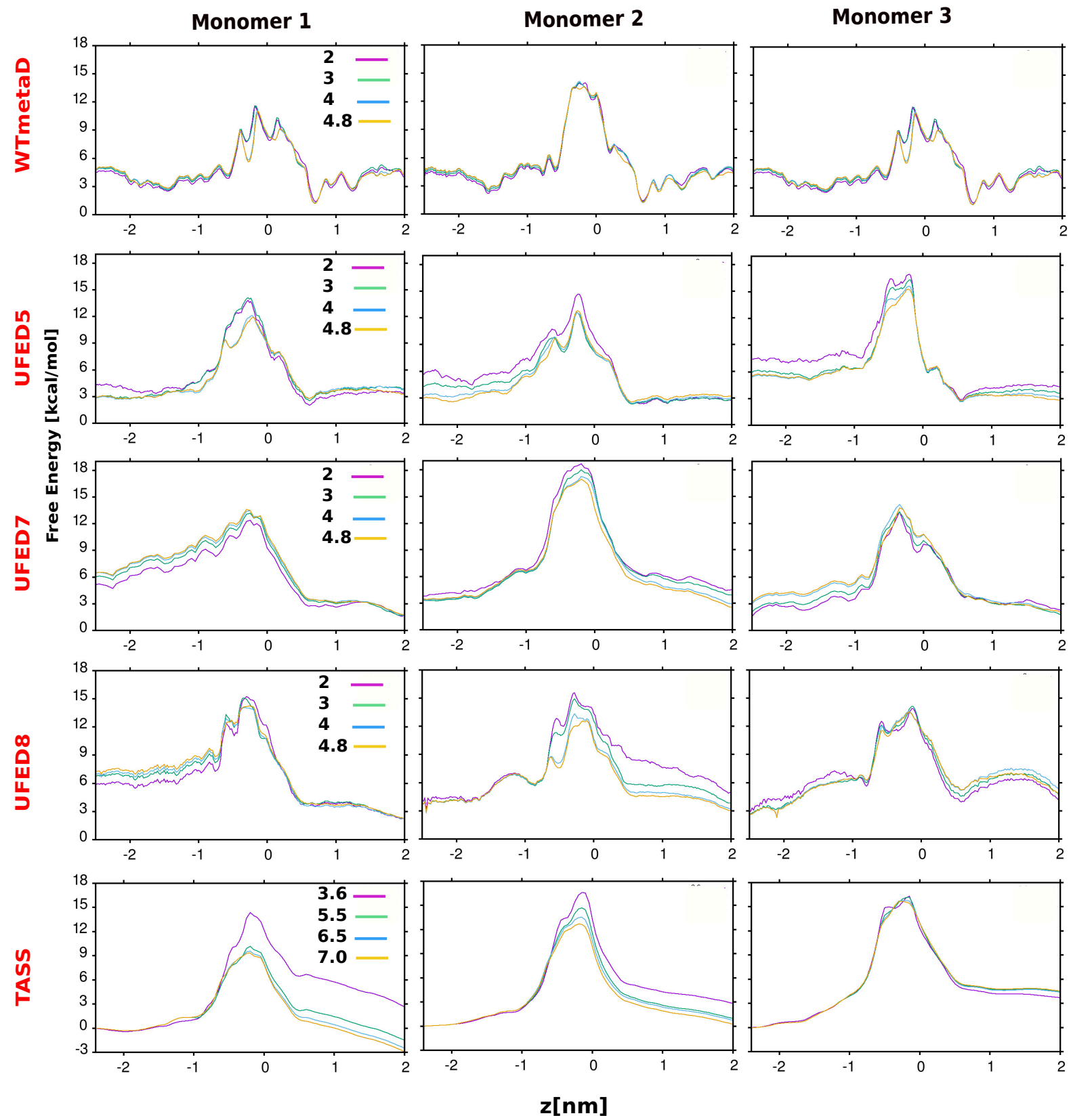

Figure S2: Time evolution of 1D FESs from independent calculations of the three monomers. As indicated by the labels, the rows correspond to the plots obtained for each simulation set with three independent simulations within each set. The numbers in legends indicate the length of the respective simulation in units of microseconds. 


\section{S3 Preliminaries and convergence for independent UFED simulations}

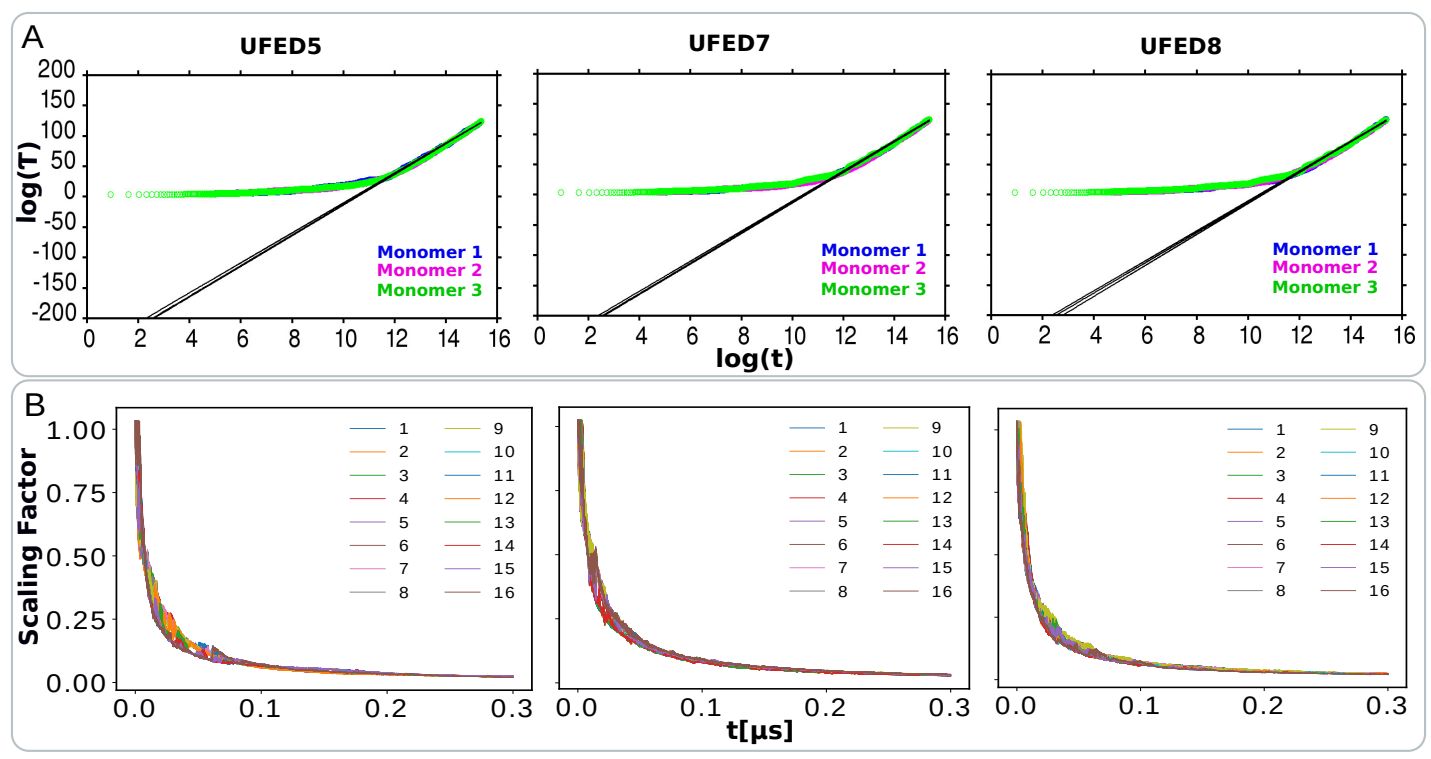

Figure S3: (A) Plot for the scaled time $\tau$ vs. simulation time $t$ on a log-log scale for the three sets of UFED simulations with 5,7 and 8 CVs. (B) The decay of the height scaling factor for the walkers in monomer 1.

The $\log (\tau)$ versus $\log (t)$ plots in Figure S3, top row, and the plots for the bias scaling factors in Figure S3, middle row, for all three sets of simulations (UFED5, UFED7 and UFED8) show that in all cases, $\log (\tau)$ attains linearity with respect to $\log (t)$, and that the bias is deposited slowly after $\approx 0.2 \mu$ s simulation time. This indicates that all simulations have achieved the quasistationary limit and thus establishes the validity of the reweighting scheme used to obtain the time-independent free energy estimates. With regard to the convergence of the independent free energy estimates obtained from UFED simulations, the time evolution of the 1D FES in Figure S2 for the three UFED simulations sets (rows 2, 3, and 4) show that the FESs were converged for most parts of the landscape in about 4 $\mu \mathrm{s}$. Finally, the evolution of the metadynamics walkers in Figure S4 shows that the walkers sample all parts of the CV space along z quite efficiently. Notably, we see a diffusive motion of the walkers with multiple crossings across the $\mathrm{CR}$ in all cases, which compared to the 
WTmetaD simulations, is a significant improvement.

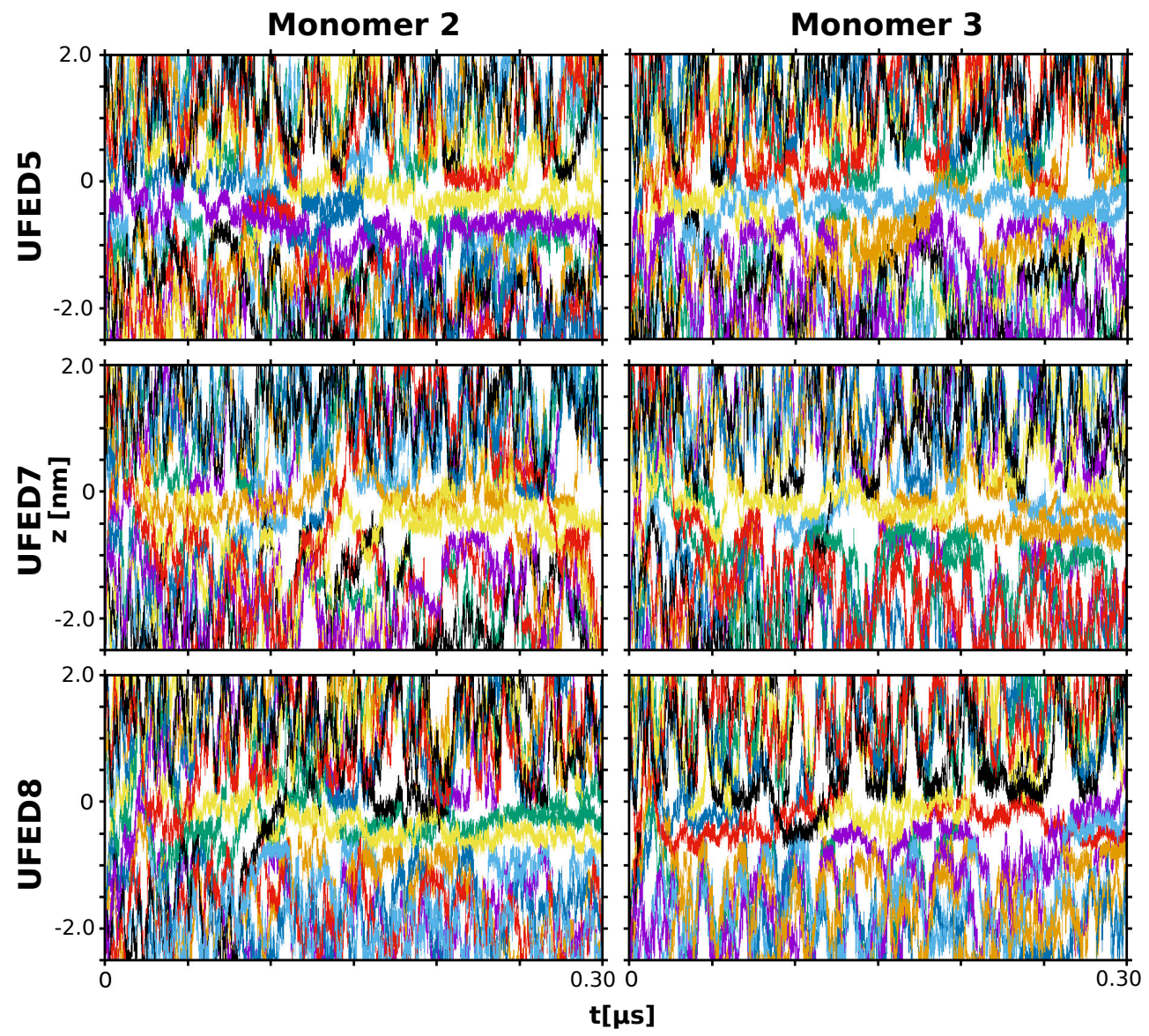

Figure S4: Time evolution of the $z$ position for each walker in the UFED simulations. The three rows correspond to the plots obtained for each simulation set with 5,7 and 8 $\mathrm{CVs}$, respectively, subject to temperature acceleration. The two columns correspond to independent simulations in monomers 2 and 3. The plots for monomer 1 are provided in the main text.

\section{S4 UFED5 simulations at $600 \mathrm{~K}$}

In the UFED simulations at $900 \mathrm{~K}$, we consistently observed conformational states wherein the ciprofloxacin molecule is buried in a groove formed by the constriction loop. In this process, this loop undergoes significant changes in conformation as shown in Figure S5. In general, the central region, i.e., $z \in[-0.5: 0.2] \mathrm{nm}$ and $z_{i j} \in[-0.2: 0.2] \mathrm{nm}$ corresponds to a sterically inaccessible region due to the presence of the constriction loop that restricts the 


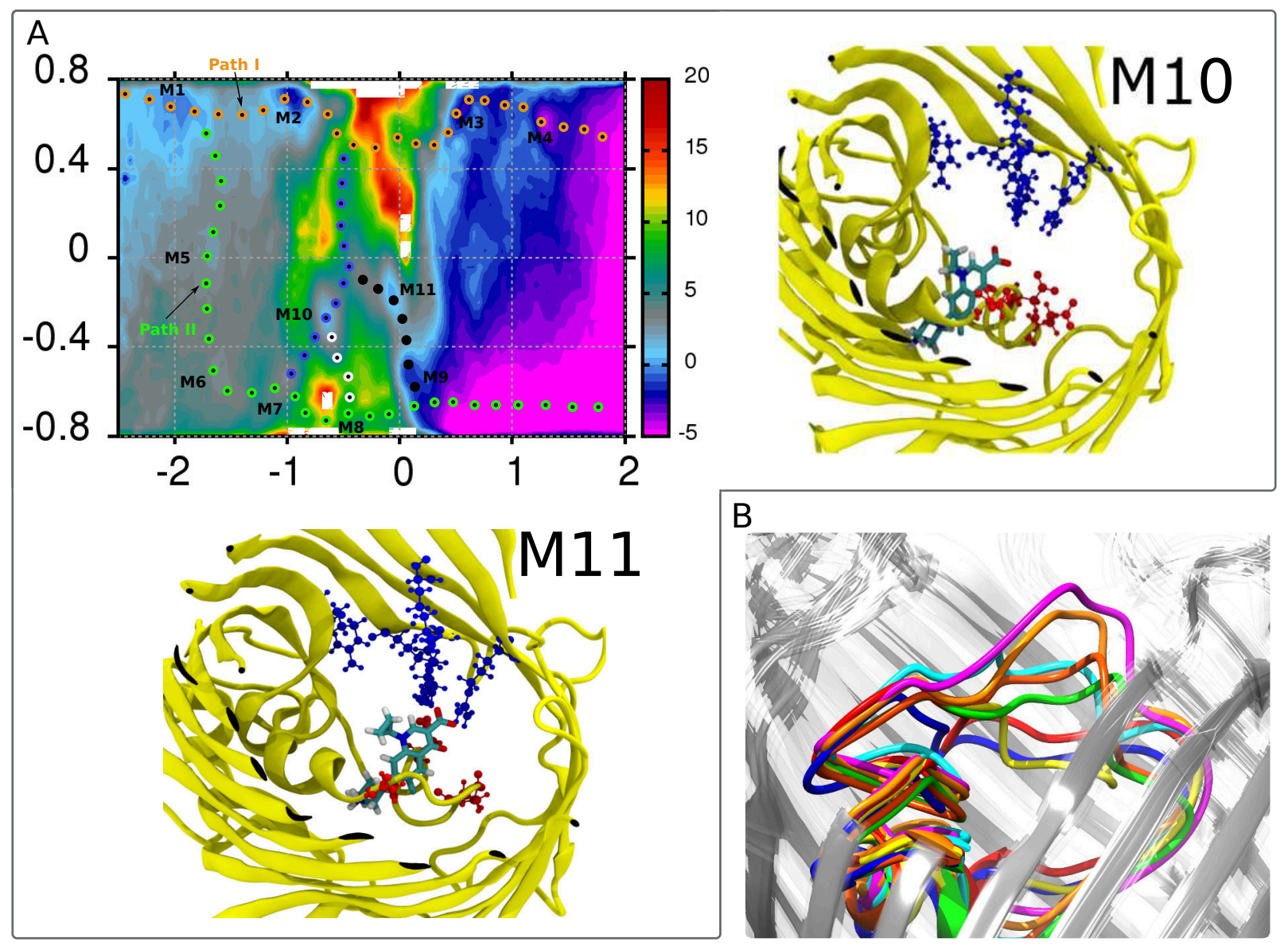

Figure S5: (A) Ciprofloxacin permeation paths through the OmpF porin obtained from UFED8 simulations. Rough outlines of the two possible paths, path I and path II, are shown in orange and green dots. Probable metastable states are labeled in black. States M10 and M11 are depicted in adjacent panels. (B) Conformational changes in the constriction loop associated with M10 and M11 states. 
rotation of bulky solutes at the CR. We reasoned that these artifacts are a result of large bias deposited at the $\mathrm{CR}$ region while the CVs are subject to temperature acceleration at $900 \mathrm{~K}$. We therefore repeated the UFED5 set with a temperature of the extended space set to $600 \mathrm{~K}$ to see if these artifacts can be avoided. In Figure S6A we still observe artifacts for monomers 1 and 3 albeit to a lesser extent. This finding suggests that using a lower temperature should ameliorate these issues. However, we also observed that reducing the temperature adversely affects sampling, especially at the barriers corresponding to the two ciprofloxacin permeation paths. Furthermore, the 1D FES plots show that the problem of non-uniform sampling of the two vestibule region still persists, as evident from the inconsistencies between free energy estimates for EC and PP regions (see Figure S6B). Thus, we concluded that a careful choice of the extended temperature and the WTmetaD Gaussian heights and bias factors is necessary to ensure correct sampling at the CR. For the present UFED simulations, we used the same height and bias factors as employed for the standard WTmetaD for a clean comparison. While the aforesaid optimizations are needed for avoiding artifacts, these do not remedy the problem of inconsistencies in sampling at the vestibules due to infrequent barrier crossing events. 

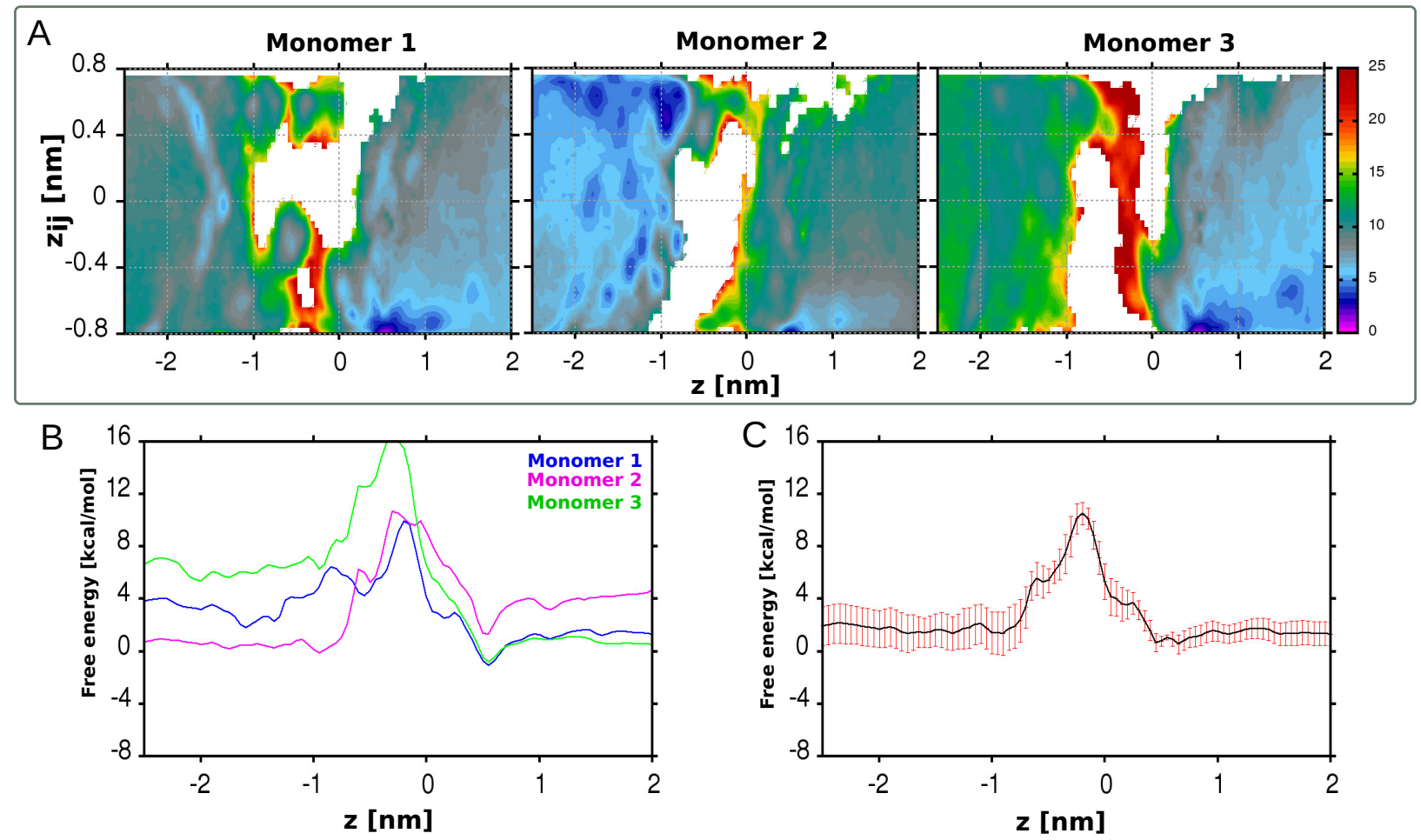

Figure S6: (A) 2D FESs for three UFED5 simulations with the extended system at $600 \mathrm{~K}$. (B) 1D FESs for three independent UFED simulations. (C) The average 1D FES and the associated standard errors from the three independent UFED runs. 


\section{S5 TASS parameters}

Umbrella sampling parameters.

Restraint Position (nm): -2.4, -2.2, -2.0, -1.8, -1.6, -1.5, -1.4, -1.3, -1.2, -1.1, -1.0, -0.9, -0.85, $-0.8,-0.75,-0.7,-0.65,-0.6,-0.55,-0.5,-0.45,-0.4,-0.35,-0.3,-0.25,-0.2,-0.15,-0.1,-0.05$, $0.0,0.1,0.2,0.3,0.4,0.5,0.6,0.7,0.8,0.9,1.0,1.2,1.4,1.6,1.8,2.0$.

$\kappa\left(\mathrm{kcal} / \mathrm{mol} / \mathrm{nm}^{2}\right): 478,478,478,478,597,717,836,836,1195,1434,1912,1912,2151$, 2270, 2390, 2509, 2509, 2629, 2629, 2748, 2629, 2390, 2390, 2390, 2270, 2151, 2151, 2151, 2151, 1912, 956, 956, 717, 836, 597, 597, 597, 717, 597, 597, 597, 597, 597, 478, 478.

TAMD parameters are provided in Table S1 below.

Table S1: Collective variables included in the sampling scheme. The same TAMD parameters were used for the UFED and TASS simulations.

\begin{tabular}{llll}
\hline CV Type & CV definition & TAMD parameters \\
\hline Solute Translation & COM distance projections & $\begin{array}{l}\kappa=10^{6} \mathrm{~kJ} \mathrm{~mol}^{-1} \mathrm{~nm}^{2} \\
\tau=0.50 \mathrm{ps} \\
\end{array}$ & \\
\hline on $x, y$ and $z$ axes & $\begin{array}{l}\kappa=5 \times 10^{5} \mathrm{~kJ} \mathrm{~mol}^{-1} \mathrm{~nm}^{2} \\
\tau=0.10 \mathrm{ps}\end{array}$ \\
\hline $\begin{array}{l}\text { Solute Rotation } \\
\text { tion }\end{array}$ & tions on $x, y$ and $z$ axes & $\begin{array}{l}\tau=10^{4} \mathrm{~kJ} \mathrm{~mol}^{-1} \mathrm{~nm}^{2} \\
\tau=0.40 \mathrm{ps}\end{array}$ \\
\hline $\begin{array}{l}\text { Solute-Lysine Interac- } \\
\text { tions }\end{array}$ & COM Distance & $\kappa=10^{5} \mathrm{~kJ} \mathrm{~mol}^{-1} \mathrm{~nm}^{2}$ \\
& & $\tau=0.50 \mathrm{ps}$ \\
\hline
\end{tabular}



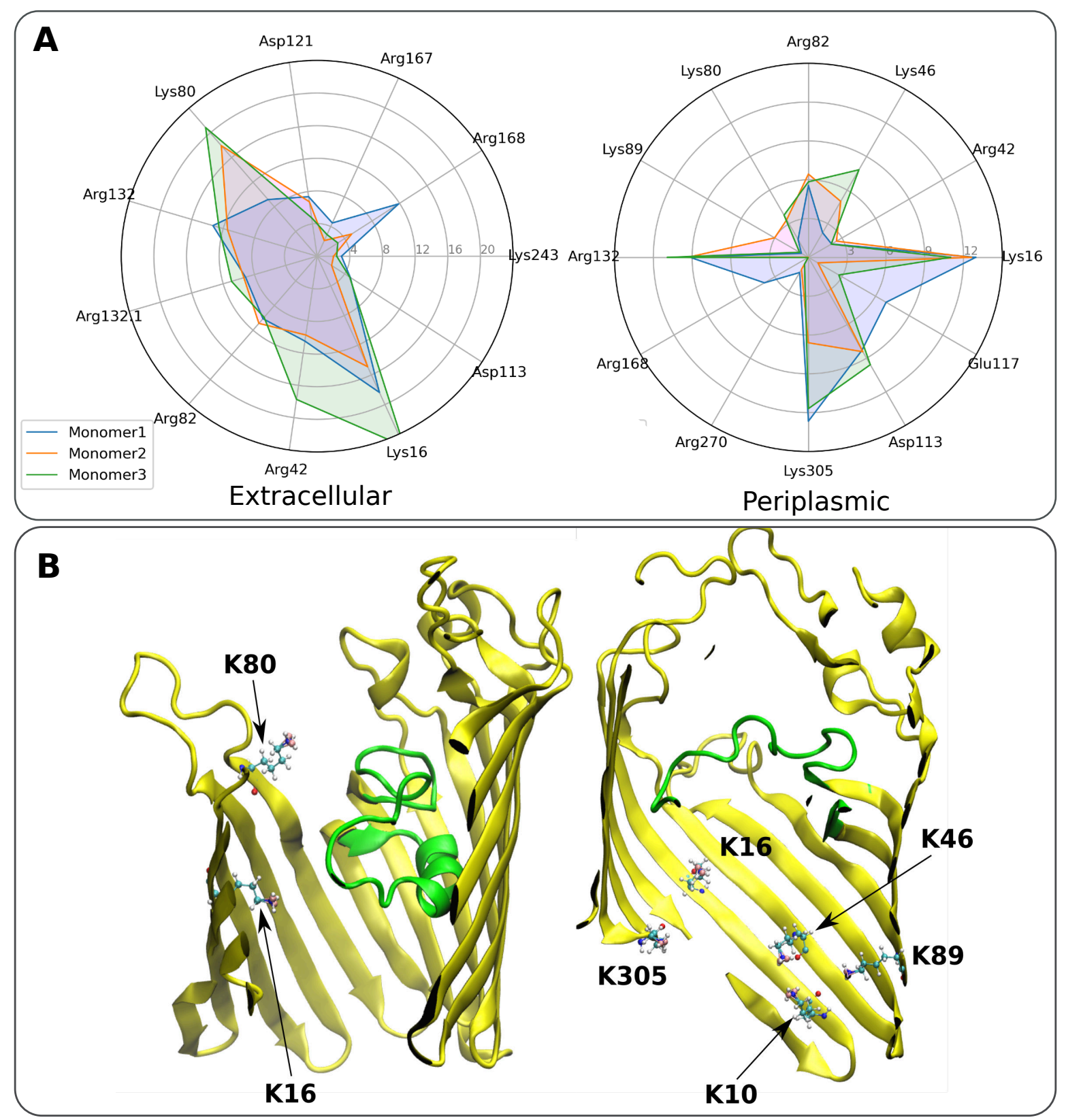

Figure S7: (A) Spider chart depicting the occupancy of hydrogen bonds between ciprofloxacin and channel residues as calculated from the TASS simulation. The radius specifies the occupancy for a given ciprofloxacin-residue pair (labeled at the periphery). Results for the three independent simulations are shown in different colors. The left panel depicts the top interactions at the extracellular region whereas the right panel depicts the same for the periplasmic region. (B) Lysine residues considered as additional CV definitions. The left panel shows lysines that are important for transport at the extracellular and constriction region. The panel on the right depicts lysines at the periplasmic vestibule. 


\section{References}

(1) Awasthi, S.; Nair, N. N. Exploring High Dimensional Free Energy Landscapes: Temperature Accelerated Sliced Sampling. J. Chem. Phys. 2017, 146, 094108.

(2) Tiwary, P.; Dama, J. F.; Parrinello, M. A Perturbative Solution to Metadynamics Ordinary Differential Equation. J. Chem. Phys. 2015, 143, 234112.

(3) Pal, A.; Pal, S.; Verma, S.; Shiga, M.; Nair, N. N. Mean Force Based Temperature Accelerated Sliced Sampling: Efficient Reconstruction of High Dimensional Free Energy Landscapes 2021, arXiv:2106.03321v1. 